Original paper

\title{
Combined PET/CT for IMRT treatment planning of NSCLC: Contrast-enhanced CT images for Monte Carlo dose calculation
}

\author{
David Mönnich $^{\mathrm{a}, *}$, Stephan Lächelt ${ }^{\mathrm{b}}$, Thomas Beyer ${ }^{\mathrm{c}, \mathrm{d}}$, Matthias K. Werner $^{\mathrm{e}}$, \\ Daniela Thorwarth ${ }^{\mathrm{a}}$ \\ ${ }^{a}$ Section for Biomedical Physics, University Hospital for Radiation Oncology, Eberhard-Karls-University Tübingen, Hoppe-Seyler-Str. 3, \\ 72076 Tübingen, Germany \\ ${ }^{\mathrm{b}}$ University Hospital for Radiation Oncology, Eberhard-Karls-University Tübingen, Germany \\ ${ }^{\mathrm{c}}$ Imaging Science Institute, Tübingen, Germany \\ ${ }^{\mathrm{d}}$ cmi-experts GmbH, Zurich, Switzerland \\ e Department of Diagnostic and Interventional Radiology, Eberhard-Karls-University Tübingen, Germany
}

\section{A R T I C L E I N F O}

\section{Article history:}

Received 24 May 2012

Received in revised form

24 July 2012

Accepted 8 August 2012

Available online 10 September 2012

\section{Keywords:}

Intravenous contrast

IMRT

Lung cancer

Monte Carlo dose calculation

\begin{abstract}
A B S T R A C T
Purpose: Combined PET/CT imaging has been proposed as an integral part of radiotherapy treatment planning (TP). Contrast-enhanced CT (ceCT) images are frequently acquired as part of the PET/CT examination to support target delineation. The aim of this dosimetric planning study was to investigate the error introduced by using a ceCT for intensity modulated radiotherapy (IMRT) TP with Monte Carlo dose calculation for non-small cell lung cancer (NSCLC).

Material and methods: Nine patients with NSCLC prior to chemo-RT were included in this retrospective study. For each patient non-enhanced, low-dose CT (neCT), ceCT and $\left[{ }^{18} \mathrm{~F}\right]$-FDG-PET emission data were acquired within a single examination. Manual contouring and TP were performed on the ceCT. An additional set of independent target volumes was auto-segmented in PET images. Dose distributions were recalculated on the neCT. Differences in dosimetric parameters were evaluated.

Results: Dose differences in PTV and lungs were small for all patients. The maximum difference in all PTVs when using ceCT images for dose calculation was $-2.1 \%$, whereas the mean difference was less than $-1.7 \%$. Maximum differences in the lungs ranged from $-1.8 \%$ to $2.1 \%$ (mean: $-0.1 \%$ ). In four patients an underestimation of the maximum spinal cord dose between $2 \%$ and $3.2 \%$ was observed, but treatment plans remained clinically acceptable.

Conclusions: Monte Carlo based IMRT planning for NSCLC patients using ceCT allows for correct dose calculation. A direct comparison to neCT-based treatment plans revealed only small dose differences. Therefore, ceCT-based TP is clinically safe as long as the maximum acceptable dose to organs at risk is not approached.
\end{abstract}

(c) 2012 Associazione Italiana di Fisica Medica. Published by Elsevier Ltd. All rights reserved.

\section{Introduction}

Combined PET/CT imaging enables the quasi-simultaneous acquisition of PET and CT data within a single examination without moving the patient off the bed in between scans [1]. Over the past decade, whole-body PET/CT has become a diagnostic modality-of-choice for the work-up of cancer patients [2]. PET/CT is increasingly employed in radio-chemotherapy treatment planning (TP) $[3,4]$. The use of PET/CT in radiotherapy (RT) treatment planning is further supported by adaptations to the hardware, the possible use of a radiotherapy pallet for patient positioning and DICOM connectivity to RT planning systems [5]. PET/CT

\footnotetext{
* Corresponding author.

E-mail address: david.moennich@med.uni-tuebingen.de (D. Mönnich).
}

examinations performed in treatment position, including positioning devices, can be directly used for tumor delineation based on the available anatomical and metabolic information [3,6,7]. Frequently, the separate acquisition of a planning CT can be waived in favor of a single PET/CT acquisition [8].

Modern radiation dose calculation algorithms rely on CTderived tissue density information. It is generally accepted that dose calculation for radiotherapy should be performed on nonenhanced CT (neCT) images acquired without intravenous (IV) contrast administration. However, combined PET/CT imaging including contrast-enhanced CT (ceCT) protocols has been shown to yield more accurate information in several tumor entities than non-enhanced PET/CT alone $[9,10]$. Further, contrast-enhanced $\mathrm{PET} / \mathrm{CT}$ facilitates clinical whole-body staging, therapy response 
assessment as well as delineation of target volumes and organs at risk (OARs) [11,12].

Modern CT contrast agents consist of iodine in a non-ionic, complexed form, for instance iopromide in different concentrations equivalent to $150-370 \mathrm{mg} / \mathrm{mL}$ ionic iodine for IV application. Ramm et al. investigated the influence of comparatively high atomic number contrast agents, such as barium-sulfate [13]. Using phantom studies, the authors found that accumulations of highdensity CT contrast agents with attenuation values of $500 \mathrm{HU}$, or less in volumes of $5 \mathrm{~cm}$ diameter, or less lead to errors in the dose calculations of $1 \%-3 \%$. Clinical patient studies reported a similarly low bias in dose calculations from the presence of IV CT contrast (300-370 mg/mL iodine) for NSCLC [14-16]. Also, some head-andneck planning studies concluded that there is no clinically relevant influence on calculated dose [16-20]. Some of these studies relied on a single CT volume only and densities within blood-vessels were virtually altered to simulate the effect of IV contrast. Even in TP of radiosurgery of cerebral arteriovenous malformations, where CT attenuation differences within the target volume were large, a significant effect was observed in selected cases only [21].

The purpose of this study was to evaluate the effect of IV CT contrast enhancement on TP with Monte Carlo (MC) dose calculation in intensity modulated radiotherapy (IMRT) of patients with non-small cell lung cancer (NSCLC). In contrast to earlier studies where artificial CT densities were assigned to single volumes, this investigation includes original neCT and ceCT images with proven co-registration, as they were acquired as part of the same PET/CT examination. In addition, the effect of IV contrast agents on dose calculation was evaluated using state-of-the art MC dose calculation, which was shown to yield accurate results for TP of NSCLC [22].

\section{Material and methods}

\section{Patient data}

Nine patients diagnosed with NSCLC prior to combined radiochemotherapy (RCT) were included in this retrospective planning study. Additional patient characteristics are summarized in Table 1. All patients were examined between 2003 and 2009 in the context of the randomized multicenter ESPATUE (Essen-Paris-Tübingen) study. Each patient recruited at the University Hospital Tübingen received a pre-treatment $\left[{ }^{18} \mathrm{~F}\right]-\mathrm{FDG}-\mathrm{PET} / \mathrm{CT}$ examination for staging purposes followed by a second PET/CT exam between day 16 and day 21 of RCT. From the originally available data $(n=34)$, a subgroup of nine patients meeting the following criteria was selected:

- Location of the main tumor mass confirmed in the upper lobe of the lung, in order to reduce respiratory motion artifacts.

- Availability of complete image volumes for neCT, ceCT and PET emission acquisition.

- Visually verified spatial alignment of neCT and ceCT image volumes using retrospective image co-registration. Here, neCT

Table 1

Characteristics of patients selected for this study $(n=9)$.

\begin{tabular}{llllcl}
\hline Pat & Age $(y)$ & Gender & TNM stage & PTV $_{\mathrm{CT}}(\mathrm{mL})$ & PTV $_{\text {PET }}(\mathrm{mL})$ \\
\hline 1 & 54 & M & T4NOM0 & 357 & 250 \\
2 & 65 & M & T4N0M0 & 881 & 213 \\
3 & 53 & M & T4N3M0 & 967 & - \\
4 & 66 & M & T1bN0M0 & 268 & 130 \\
5 & 56 & M & T2bNOM0 & 1002 & 267 \\
6 & 63 & M & T4N2M0 & 978 & 329 \\
7 & 52 & M & T4NOM0 & 994 & 581 \\
8 & 57 & M & T3N0M0 & 584 & 173 \\
9 & 54 & F & T2aN2M0 & 480 & 92 \\
\hline
\end{tabular}

and ceCT image volumes were aligned using automated linear and non-linear co-registration based on mutual information algorithms (Mirada XD3, Mirada Medical Ltd., UK). Following the spatial co-registration, the image registration quality was visually assessed and then the image volume alignment of neCT and ceCT data was confirmed. If the residual maximum average misregistration vector provided by the software was less than the PET voxel size ( $600 \mathrm{~mm}, 128$ matrix size: $4.7 \mathrm{~mm}$ ) then the CT image volumes were considered accurately aligned for the purpose of CT-based attenuation correction and dose planning. Note, the spatial resolution of the CT and PET emission data must be the same.

\section{Patient protocol}

Patients fasted for $6 \mathrm{~h}$ prior to the injection of $\left[{ }^{18} \mathrm{~F}\right]-\mathrm{FDG}$. Patients were then injected with a median $\left[{ }^{18} \mathrm{~F}\right]-\mathrm{FDG}$ dose of $441 \mathrm{MBq}$. During the median uptake phase of 62 min patients rested in a reclined position in a dimly lit room. A negative oral CT contrast was administered during the uptake phase [23]. Patients were positioned head first supine on a vacuum cushion and an RT table top with their arms raised and supported above the head. PET/CT imaging was performed on a Biograph HiRez 16 PET/CT (Siemens Healthcare, Germany).

The imaging sequence was as follows: topogram scan, low-dose non-enhanced CT (neCT), arterial-phase contrast-enhanced CT (ceCT) following $100 \mathrm{~mL}$ of $370 \mathrm{mg} / \mathrm{mL}$ iodine-equivalent non-ionic intravenous iodinated contrast agent (Ultravist 370, BayerSchering, Germany) and a multi-bed emission scan of $3 \mathrm{~min} /$ bed. More details are given in [24]. Emission and CT imaging with and without IV contrast was performed during tidal breathing and in mid-expiration, respectively [25]. PET image reconstruction was performed using a 2D OSEM algorithm with 2 iterations, 8 subsets and a matrix size of $128 \times 128$. PET attenuation correction was performed based on the neCT images with a well-established algorithm described elsewhere [26].

\section{Treatment planning and target volumes}

For this retrospective study PET/CT image data from the pretreatment examination were used. Target volumes (TV) and OARs were delineated manually on the ceCT using the treatment planning system Oncentra MasterPlan (Nucletron, The Netherlands). A clinical target volume $\left(\mathrm{CTV}_{\mathrm{CT}}\right)$ including the primary tumor and involved lymph nodes was created by an experienced RT specialist in accordance with the guidelines of the International Commission on Radiation Units \& Measurements (ICRU) Report 62 [27]. A second clinical target volume $\left(\mathrm{CTV}_{\mathrm{PET}}\right)$ was automatically segmented in the PET images using a fixed threshold at $42 \%$ of the maximum standardized uptake value [28] (Siemens TrueD, Siemens Healthcare). Both volumes were uniformly expanded by a $1.5 \mathrm{~cm}$ safety margin to obtain planning target volumes $\left(\mathrm{PTV}_{\mathrm{CT}}\right.$ and PTV $\mathrm{PET}_{\mathrm{P}}$, Fig. 1) that were subject to a dose prescription of $60 \mathrm{~Gy}$ in 30 fractions. OARs considered for NSCLC IMRT planning were heart, total lung, and spinal cord.

IMRT TP was performed on the ceCT with the Hyperion planning system using a dedicated MC dose engine [22,29]. The voxel size for the dose calculation and estimated MC accuracy was set to $3 \mathrm{~mm}$ and $2 \%$, respectively. Six to ten coplanar $6 \mathrm{MV}$ beams were applied. The primary objective was to enclose the PTV within the $95 \%$ isodose line as long as normal tissue constraints were met and to achieve high target dose uniformity. Doses higher than $105 \%$ of the prescribed dose were not allowed. 


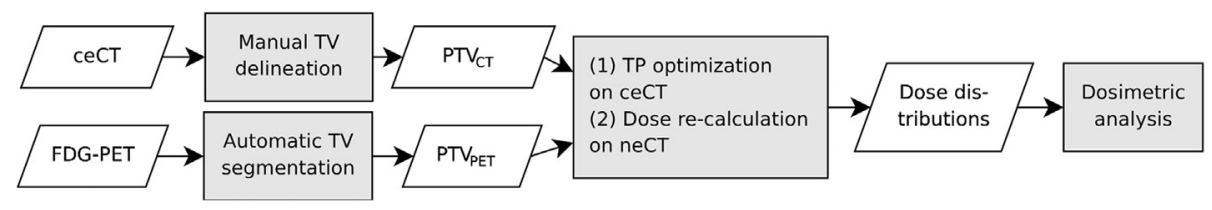

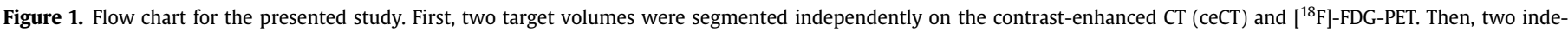

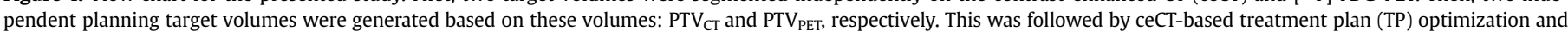
dose recalculation on the corresponding non-enhanced low-dose CT (neCT). Finally, the resulting differences in dosimetric parameters were evaluated.

In a second step, the created treatment plans were recalculated based on tissue densities in the neCT (Fig. 1). Here, accurate alignment of neCT and ceCT image volumes was assumed, since the patients eligible for this study were shown not to have moved in between the CT examinations. Previously, it was reported that dose errors induced by differences in attenuation values between lowand full-dose CTs are below 2\% [30]. Thus, it is legitimate to use doses calculated on non-enhanced low-dose CTs as references.

\section{Data analysis}

Relative dose differences in the PTV, the spinal cord and healthy lung tissue (total lung - PTV) were calculated as

$\Delta D=\left(D_{\text {cect }}-D_{\text {neCT }}\right) / D_{\text {cect }} \times 100$

where $\Delta D$ is the relative dose difference in \% and $D$ is the respective dosimetric parameter. This definition differs in sign compared to results in [15]. Relative differences were calculated for mean and maximum dose. Maximum dose differences of more than $3 \%$ were considered to be clinically relevant. Additionally, differences in the fractional volume of healthy lung tissue receiving more than $20 \mathrm{~Gy}$ $\left(V_{20}\right)$ and the minimum dose within 95\% of the PTV $\left(D_{95}\right)$ were evaluated. PTV $\mathrm{V}_{\text {PET }}$ plans were assessed independently using the same method.

\section{Results}

A direct comparison of the CT attenuation values in ne- and ceCT images demonstrated that IV contrast leads to an increased fraction of higher attenuating tissue voxels in relevant sub-volumes (Fig. 2). Contrast administration leads to an increased number of voxel values between $150 \mathrm{HU}$ and $300 \mathrm{HU}$ in the ceCT.

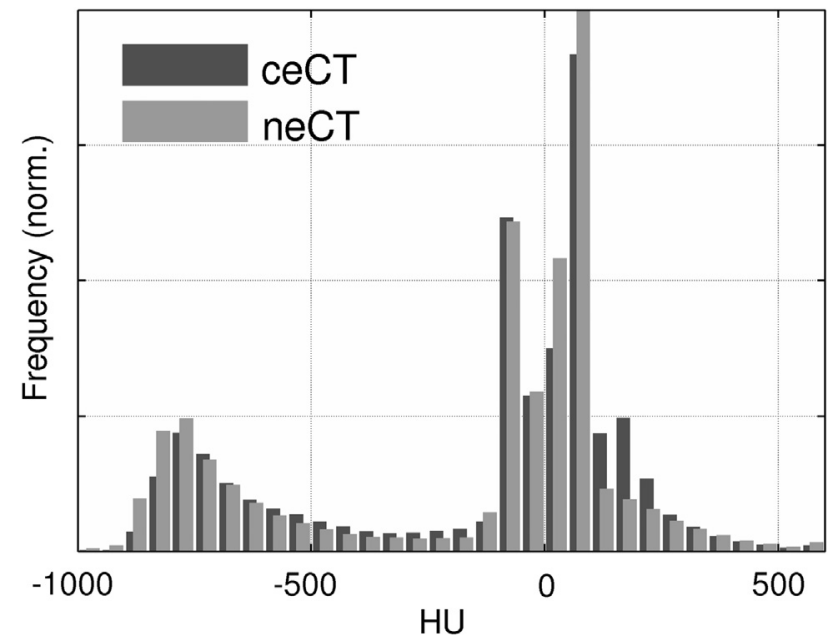

Figure 2. Histogram of Hounsfield units in the non-enhanced (neCT) and contrastenhanced (ceCT) CT volume covering the co-axial range of the target volume (patient 5). Note the increased number of voxels with higher attenuation values in the range of 150-300 $\mathrm{HU}$ after intravenous contrast administration (ceCT).
Manually delineated PTVs had a mean size of $723 \mathrm{~mL}$ (range: $268 \mathrm{~mL}-1002 \mathrm{~mL}$ ), and were generally larger than those segmented from PET images with a mean volume of $254 \mathrm{~mL}$ (range: $92 \mathrm{~mL}-581 \mathrm{~mL}$ ) (Table 1 ).

An acceptable dose coverage complying with normal tissue constraints was achieved in all treatment plans with one exception where the full dose was applicable to the manually delineated CTV only (patient 10). The resulting median mean dose was $59.3 \mathrm{~Gy}$ (range: $55.2 \mathrm{~Gy}-60.9 \mathrm{~Gy}$ ) for PTV $\mathrm{CT}_{\text {T }}$ plans and $59.8 \mathrm{~Gy}$ (59.2 Gy$60.7 \mathrm{~Gy}$ ) for PTV $\mathrm{PET}_{\mathrm{P}}$ plans. Dose parameters for all plans created on the neCT images are shown in Table 2.

Table 3 shows the relative dose differences $\Delta D$ between neCT and ceCT for PTVs as well as OARs. Relative dose differences in the target volumes were below $2 \%$ in almost all cases. For patient 9 , differences in the maximum dose and $D_{95}$ for the PTV were $-2.1 \%$ and $-2.2 \%$, respectively, which reflects a noncritical overdosage in the original plan. In contrast, a positive difference of $2.6 \%$ in $D_{95}$ in the PET-PTV plan for the same patient reveals a moderate underdosage.

For the mean PTV dose mainly negative differences were found with a median value for $\mathrm{PTV}_{\mathrm{CT}}$ and $\mathrm{PTV}_{\mathrm{PET}}$ of $-0.9 \%$, and $-0.3 \%$, respectively. This indicates a small systematic underestimation of the applied dose caused by the usage of the ceCT as a basis for dose calculation. Dose differences in the lung were below $2 \%$ with the exception in patient 9 , where $V_{20}$ and mean dose were underestimated by $-3.6 \%$ and $-2.4 \%$, respectively. Differences in mean spinal cord doses were below $2 \%$ for all plans with median values of $-1.2 \%\left(\mathrm{PTV}_{\mathrm{CT}}\right)$ and $-1.5 \%$ ( $\left.\mathrm{PTV}_{\mathrm{PET}}\right)$. Maximum spinal cord doses were underestimated in all but one case. In some plans the underestimation was larger than $2 \%$ with a maximum of $-3.2 \%$ in the PTV observed in this particular case, considering that absolute maximum doses remained below $45 \mathrm{~Gy}$.

Figure 3 shows IMRT treatment plans on neCT and ceCT images for patients 9 and 5. Examples of the resulting dose distributions are depicted for selected axial image planes. In patient 9, differences between the original and the recalculated dose distribution (Fig. 3a, b) can be identified in a region where the PTV $\mathrm{CT}_{\text {T }}$ overlaps with large arteries containing contrast agent. The shape of the 95\% isodose line is different and a small overdosed region (>63 Gy) appears in the recalculated plan. In another patient (patient 5 , Fig. 3d, e) the isodose lines of both dose distributions are virtually

Table 2

Characteristics of treatment plans created on non-enhanced CT volumes for the patient group of this study.

\begin{tabular}{llc}
\hline & \multicolumn{2}{l}{ Dosimetric parameter median (range) } \\
\cline { 2 - 3 } & PTV $_{\mathrm{CT}}$ & PTV $_{\mathrm{PET}}$ \\
\hline Mean dose PTV $(\mathrm{Gy})$ & $59.3(55.2-60.9)$ & $59.8(59.2-60.7)$ \\
Max dose PTV (Gy) & $65.4(64.3-67.0)$ & $64.7(62.7-65.9)$ \\
$D_{95}(\mathrm{~Gy})$ & $56.3(47.5-58.2)$ & $56.6(55.9-57.8)$ \\
Mean dose lungs $(\mathrm{Gy})$ & $19.8(9.5-23.7)$ & $10.8(7.1-16.9)$ \\
Max dose lungs $(\mathrm{Gy})$ & $64.2(63.3-66.8)$ & $63.7(62.7-65.9)$ \\
$V_{20}(\%)$ & $25.9(13.7-34.3)$ & $16.3(6.3-25.4)$ \\
Mean dose spinal cord $(\mathrm{Gy})$ & $12.3(7.3-18.2)$ & $6.9(5.5-11.9)$ \\
Max dose spinal cord $(\mathrm{Gy})$ & $36.2(21.7-40.6)$ & $25.6(17.1-46.5)$ \\
\hline
\end{tabular}


Table 3

Relative differences for various dosimetric parameters (Equation (1)). Values larger than $2 \%$ are marked in gray. PET data were unavailable for patient 3.

\begin{tabular}{|c|c|c|c|c|c|c|c|c|c|c|c|c|c|c|c|c|}
\hline \multirow[t]{3}{*}{ Pat \# } & \multicolumn{8}{|l|}{$\mathrm{PTV}_{\mathrm{CT}}$} & \multicolumn{8}{|l|}{ PTV $_{\text {PET }}$} \\
\hline & \multicolumn{3}{|l|}{ PTV } & \multicolumn{3}{|c|}{ Total lung } & \multicolumn{2}{|c|}{ Spinal cord } & \multicolumn{3}{|c|}{ PET-PTV } & \multicolumn{3}{|c|}{ Total lung } & \multicolumn{2}{|c|}{ Spinal cord } \\
\hline & Mean & Max & $D_{95}$ & Mean & Max & $V_{20}$ & Mean & Max & Mean & Max & $D_{95}$ & Mean & Max & $V_{20}$ & Mean & Max \\
\hline 1 & $-0.1 \%$ & $0.0 \%$ & $0.2 \%$ & $-0.8 \%$ & $-0.8 \%$ & $-0.6 \%$ & $0.7 \%$ & $0.0 \%$ & $0.2 \%$ & $0.5 \%$ & $0.3 \%$ & $-0.6 \%$ & $1.8 \%$ & $0.0 \%$ & $-0.8 \%$ & $-1.5 \%$ \\
\hline 2 & $-0.7 \%$ & $-0.6 \%$ & $-0.7 \%$ & $-0.9 \%$ & $-1.2 \%$ & $-1.1 \%$ & $-1.4 \%$ & $-1.9 \%$ & $-0.4 \%$ & $0.7 \%$ & $0.0 \%$ & $-0.6 \%$ & $-0.1 \%$ & $-0.2 \%$ & $-1.5 \%$ & $-1.7 \%$ \\
\hline 3 & $-0.9 \%$ & $-0.1 \%$ & $-0.7 \%$ & $-0.9 \%$ & $-0.3 \%$ & $-0.8 \%$ & $-1.0 \%$ & $-0.2 \%$ & & & & & & & & \\
\hline 4 & $-1.4 \%$ & $-0.9 \%$ & $-0.4 \%$ & $-1.2 \%$ & $2.1 \%$ & $-1.2 \%$ & $-1.2 \%$ & $-1.7 \%$ & $0.1 \%$ & $0.4 \%$ & $1.1 \%$ & $-1.1 \%$ & $0.1 \%$ & $-1.0 \%$ & $-1.6 \%$ & $-2.5 \%$ \\
\hline 5 & $-1.3 \%$ & $-1.8 \%$ & $-1.4 \%$ & $-1.2 \%$ & $-0.3 \%$ & $-1.5 \%$ & $-1.3 \%$ & $-0.9 \%$ & $-1.2 \%$ & $-1.6 \%$ & $-0.8 \%$ & $-1.1 \%$ & $0.0 \%$ & $-1.7 \%$ & $-1.5 \%$ & $-3.2 \%$ \\
\hline 6 & $-1.0 \%$ & $-0.2 \%$ & $-1.3 \%$ & $-1.2 \%$ & $-0.2 \%$ & $-1.2 \%$ & $-1.3 \%$ & $-2.2 \%$ & $-0.8 \%$ & $-1.0 \%$ & $-1.2 \%$ & $-1.2 \%$ & $-1.3 \%$ & $-1.1 \%$ & $-1.1 \%$ & $-1.0 \%$ \\
\hline 7 & $-0.4 \%$ & $-0.2 \%$ & $-0.2 \%$ & $-1.3 \%$ & $1.0 \%$ & $-1.4 \%$ & $-1.3 \%$ & $-2.1 \%$ & $-0.6 \%$ & $-0.1 \%$ & $-0.3 \%$ & $-1.7 \%$ & $-1.8 \%$ & $-1.2 \%$ & $-2.0 \%$ & $-3.0 \%$ \\
\hline 8 & $-0.6 \%$ & $0.2 \%$ & $-0.5 \%$ & $-1.1 \%$ & $-0.1 \%$ & $-1.6 \%$ & $-1.0 \%$ & $0.4 \%$ & $-0.3 \%$ & $-1.4 \%$ & $0.2 \%$ & $-1.0 \%$ & $0.9 \%$ & $-0.6 \%$ & $-0.5 \%$ & $-1.8 \%$ \\
\hline 9 & $-1.7 \%$ & $-2.1 \%$ & $-2.2 \%$ & $-2.4 \%$ & $0.7 \%$ & $-3.6 \%$ & $-0.6 \%$ & $-1.5 \%$ & $0.0 \%$ & $0.7 \%$ & $2.6 \%$ & $-2.6 \%$ & $1.2 \%$ & $-1.5 \%$ & $-1.9 \%$ & $-2.0 \%$ \\
\hline Median & $-0.9 \%$ & $-0.2 \%$ & $-0.7 \%$ & $-1.2 \%$ & $-0.2 \%$ & $-1.2 \%$ & $-1.2 \%$ & $-1.5 \%$ & $-0.3 \%$ & $0.1 \%$ & $0.1 \%$ & $-1.1 \%$ & $0.0 \%$ & $-1.1 \%$ & $-1.5 \%$ & $-1.9 \%$ \\
\hline
\end{tabular}

identical. Voxel-based relative dose differences reveal some mismatch artifacts close to the body surface and also local differences smaller than $-6 \%$ and larger than $6 \%$ in low-dose regions (Fig. 3c, f). Figure 4 shows the corresponding dose volume histograms (DVH) for patients 9 and 5. Here, the effect of ceCT-based TP, which is a slight underestimation in terms of dose in the contrastenhanced target regions, is clearly visible.

\section{Discussion}

The uniqueness of the presented study is given by (a) the availability of pairs of original NSCLC CT image data acquired with and without IV contrast in one single examination and (b) by the use of an MC dose calculation to investigate the effect of ceCT on RT
TP. As neCT and ceCT were acquired during the same examination and in the same breathing phase, accurate co-registration of the corresponding image data sets could be validated retrospectively. As a result, identical regions of interest could be used for ne- and ceCT-based TP. Furthermore, the type of dose calculation used to study this effect is of major importance. It is known, that current analytical dose calculation algorithms introduce errors of up to $5 \%$, especially in the case of high density materials and high density gradients, i.e. tissue-bone and tissue-air interfaces [31,32]. It is not safe to assume that results obtained with such algorithms are as well applicable to more accurate MC dose calculation.

We were able to demonstrate that dose calculation based on ceCT images is possible and results in acceptable treatment plans. Errors introduced by using CT images following IV contrast

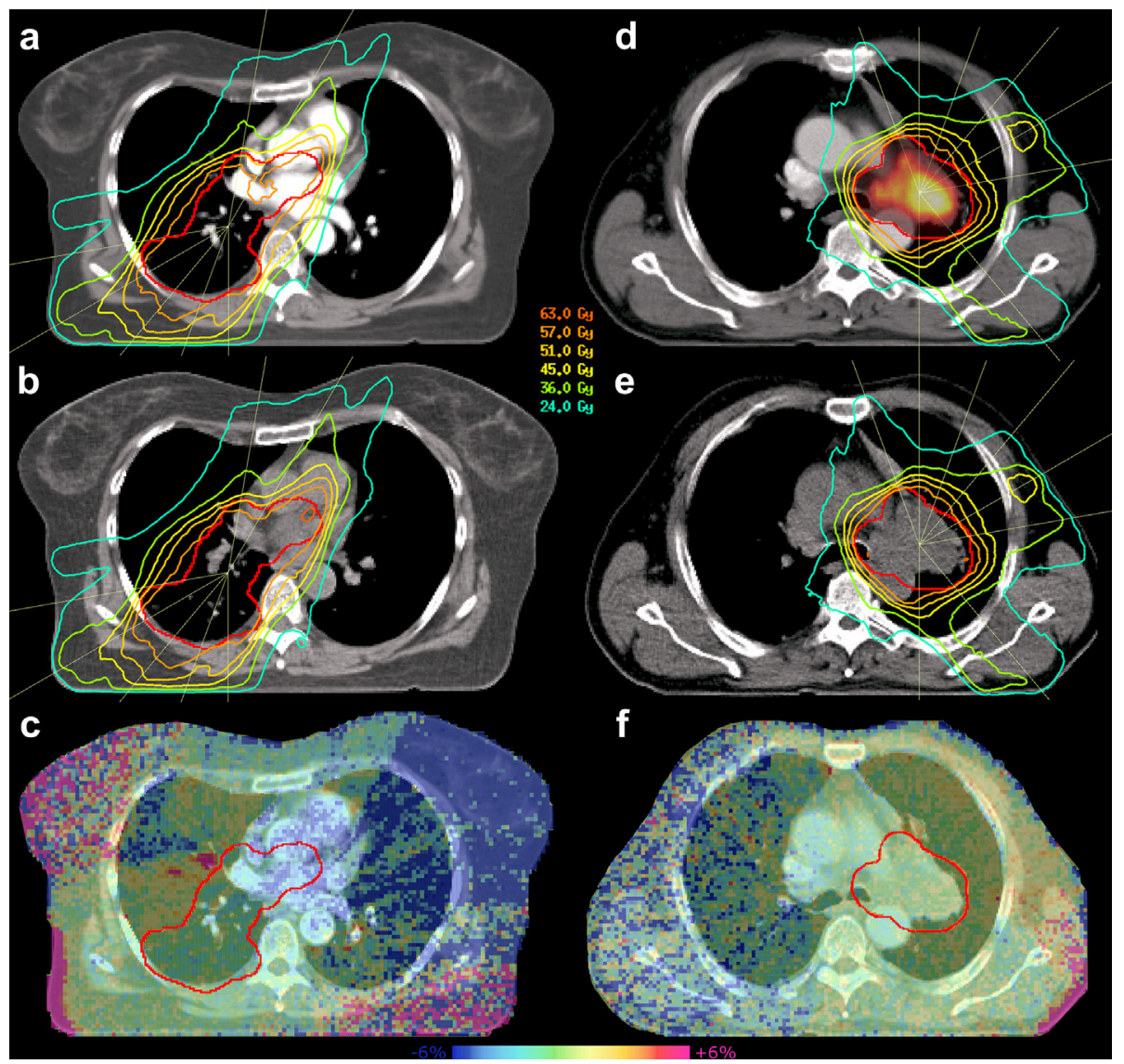

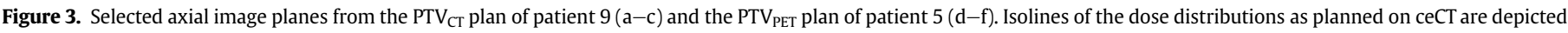

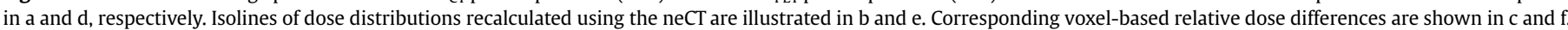



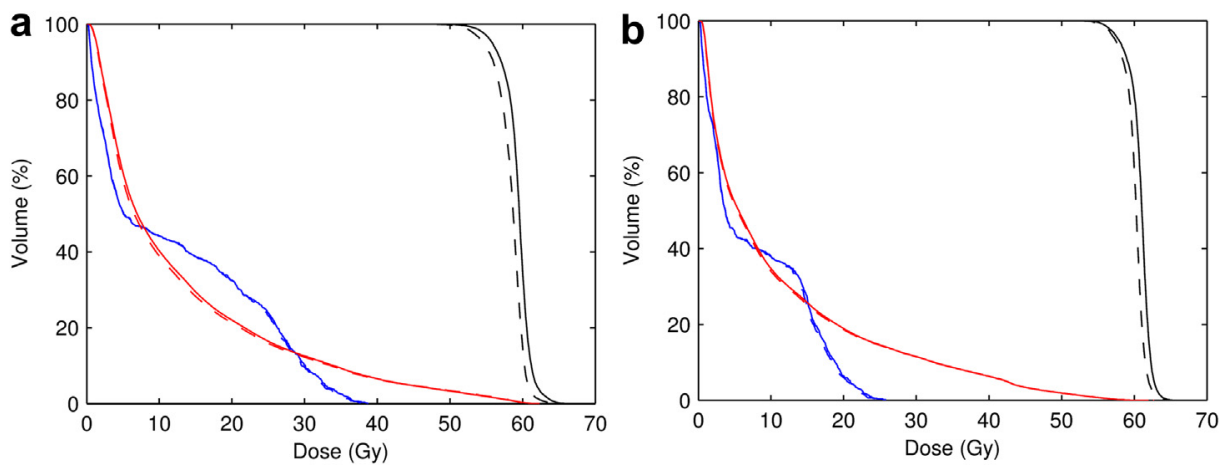

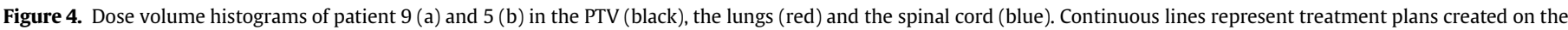

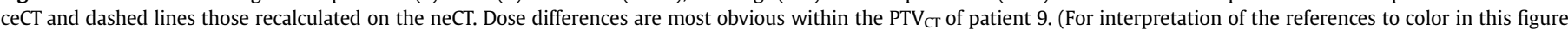
legend, the reader is referred to the web version of this article.)

enhancement were clinically negligible in all patients. In most cases the applied dose in targets as well as OARs was underestimated. Underdosage effects were regional and resulted either from the attenuation of beams by enhanced structures shadowing a volumeof-interest, or from the direct effect of less dose being deposited in enhanced structures compared to non-enhanced CT TP.

The magnitude of dose differences in PTVs and healthy lung tissue were much smaller than errors anticipated from tumor motion during irradiation (Table 3). Specifically, the dose simulation underestimates target doses, which may translate into excess radiation doses. This increase may, however, be acceptable in NSCLC if it is below $110 \%$. This result agrees with other studies investigating the influence of intravenous contrast on IMRT planning of NSCLC $[14,15]$.

Dose differences in the lung, though much larger than reported by Xiao et al. [15], were clinically irrelevant (Table 3). Deviations from the results reported by Xiao et al. [15] may be due to the fact that in their study a convolution/superposition algorithm was used for dose calculation. Good agreement with the results of Shi et al. [14] was found in the patient group averages of differences in $V_{20}$ and mean lung dose. Generally, it is difficult to compare the presented patient specific values with other studies, because most of them reported group averages only, thus obliterating extreme differences from the results.

Relevant differences were found in the maximum spinal cord dose, which varied by up to $3.2 \%$ on a single patient basis. These patient based differences are considerably larger than the cohort averages of $0.4 \%$ as reported by Shi et al. using single CTs and density override [14]. In the spinal cord, errors of the observed magnitude might be relevant in cases with high local dose burdens approaching $45 \mathrm{~Gy}$. In these cases it may be feasible to adjust tissue densities following the delineation of contrast-enhanced regions.

A careful clinical interpretation of the dosimetric differences in patients of this pilot study is required. First, the minor differences can be explained by the use of an MC dose engine and two independent CT image volumes (neCT and ceCT). The observed dose differences may be caused also by effects of respiratory motion, which may lead to sub-cm, or even sub-mm alignment accuracies of the lesions on neCT and ceCT. Here, the neCT and ceCT image volumes were acquired sequentially in mid-expiration breath-hold [24,25], which improves spatial co-registration of complementary image volumes [33].

In addition to the small group size of this study, it should be mentioned that a potential influence of dose calculation errors on the convergence of the TP software to the optimum treatment plan was not investigated in this study.

\section{Conclusions}

Our data support the notion of using contrast-enhanced CT as part of combined PET/CT imaging for radiotherapy treatment planning in NSCLC without the need to acquire a separate, low-dose CT. MC dose calculation based on ceCT images was shown to yield correct treatment plans in terms of relative errors in dose compared to neCT-based planning. Care must be taken in cases with perceived high doses to OARs, where critical overdosages might result when TP is performed on a contrast-enhanced CT.

\section{Conflict of interest}

Thomas Beyer is founder and president of cmi-experts $\mathrm{GmbH}$, Zurich and reports no conflict of interest.

\section{Acknowledgments}

DT is supported by the European Social Fund and the Ministry of Science, Research and the Arts Baden-Württemberg. We thank Markus Alber (University Hospital for Radiation Oncology, Tübingen) for providing us with a research version of the TPS Hyperion and Mirada Medical, UK for XD3 image fusion software.

\section{List of abbreviations}

$\begin{array}{ll}\text { ceCT } & \text { contrast-enhanced computed tomography } \\ \text { CTV } & \text { clinical target volume } \\ \text { DVH } & \text { dose volume histogram } \\ \text { FDG } & \text { fluorodesoxyglucose } \\ \text { HU } & \text { Hounsfiled Unit } \\ \text { ICRU } & \text { International Commission for Radiation Units \& } \\ & \text { Measurement } \\ \text { IMRT } & \text { intensity modulated radiation therapy } \\ \text { IV } & \text { intravenous } \\ \text { MC } & \text { Monte Carlo } \\ \text { neCT } & \text { non-enhanced computed tomography } \\ \text { NSCLC } & \text { non-small cell lung cancer } \\ \text { OAR } & \text { organ at risk } \\ \text { OSEM } & \text { ordered subsets expectation maximization } \\ \text { PET/CT } & \text { positron emission tomography/computed tomography } \\ \text { PTV } & \text { planning target volume } \\ \text { RCT } & \text { radio-chemotherapy } \\ \text { RT } & \text { radiation therapy } \\ \text { TP } & \text { treatment planning } \\ \text { TV } & \text { target volume }\end{array}$




\section{References}

[1] Beyer T, Townsend DW, Brun T, Kinahan PE, Charron M, Roddy R, et al. A combined PET/CT scanner for clinical oncology. J Nucl Med 2000;41: 1369-79.

[2] Czernin J, Allen-Auerbach M, Schelbert HR. Improvements in cancer staging with PET/CT: literature-based evidence as of September 2006. J Nucl Med 2007;48(Suppl. 1):78S-88S

[3] Nestle U, Weber W, Hentschel M, Grosu AL. Biological imaging in radiation therapy: role of positron emission tomography. Phys Med Biol 2009;54:R1-25.

[4] Lammering G, De Ruysscher D, van Baardwijk A, Baumert BG, Borger J, Lutgens L, et al. The use of FDG-PET to target tumors by radiotherapy. Strahlenther Onkol 2010;186:471-81.

[5] Sattler B, Lee JA, Lonsdale M, Coche E. PET/CT (and CT) instrumentation, image reconstruction and data transfer for radiotherapy planning. Radiother Oncol 2010;96:288-97.

[6] Evans PM. Anatomical imaging for radiotherapy. Phys Med Biol 2008;53: R151-91.

[7] Thorwarth D, Schaefer A. Functional target volume delineation for radiation therapy on the basis of positron emission tomography and the correlation with histopathology. Q J Nucl Med Mol Imaging 2010;54: 490-9.

[8] Thorwarth D, Geets X, Paiusco M. Physical radiotherapy treatment planning based on functional PET/CT data. Radiother Oncol 2010;96:317-24.

[9] Pfannenberg AC, Aschoff P, Brechtel K, Müller M, Bares R, Paulsen F, et al. Low dose non-enhanced $C T$ versus standard dose contrast-enhanced $C T$ in combined PET/CT protocols for staging and therapy planning in non-small cell lung cancer. Eur J Nucl Med Mol Imaging 2007;34:36-44.

[10] Antoch G, Freudenberg LS, Beyer T, Bockisch A, Debatin JF. To enhance or not to enhance? $18 \mathrm{~F}-\mathrm{FDG}$ and CT contrast agents in dual-modality 18F-FDG PET/ CT. J Nucl Med 2004;45(Suppl. 1):56S-65S.

[11] De Ruysscher D, Kirsch C-M. PET scans in radiotherapy planning of lung cancer. Radiother Oncol 2010;96:335-8.

[12] Roy AEF, Wells P. Volume definition in radiotherapy planning for lung cancer: how the radiologist can help. Cancer Imaging 2006;6:116-23.

[13] Ramm U, Damrau M, Mose S, Manegold K-H, Rahl CG, Böttcher H-D. Influence of CT contrast agents on dose calculations in a 3D treatment planning system. Phys Med Biol 2001:46:2631-5.

[14] Shi W, Liu C, Lu B, Yeung A, Newlin HE, Amdur RJ, et al. The effect of intravenous contrast on photon radiation therapy dose calculations for lung cancer. Am J Clin Oncol 2010;33:153-6.

[15] Xiao J, Zhang H, Gong Y, Fu Y, Tang B, Wang S, et al. Feasibility of using intravenous contrast-enhanced computed tomography (CT) scans in lung cancer treatment planning. Radiother Oncol 2010;96:73-7.

[16] Shibamoto Y, Naruse A, Fukuma H, Ayakawa S, Sugie C, Tomita N. Influence of contrast materials on dose calculation in radiotherapy planning using computed tomography for tumours at various anatomical regions: a prospective study. Radiother Oncol 2007;84:52-5.

[17] Liauw SL, Amdur RJ, Mendenhall WM, Palta J, Kim S. The effect of intravenous contrast on intensity-modulated radiation therapy dose calculations for head and neck cancer. Am J Clin Oncol 2005;28:456-9.
[18] Létourneau D, Finlay M, O’Sullivan B, Waldron JN, Cummings BJ, Ringash J, et al. Lack of influence of intravenous contrast on head and neck IMRT dose distributions. Acta Oncol 2008;47:90-4.

[19] Choi Y, Kim J-K, Lee H-S, Hur W-J, Hong Y-S, Park S, et al. Influence of intravenous contrast agent on dose calculations of intensity modulated radiation therapy plans for head and neck cancer. Radiother Oncol 2006;81:158-62.

[20] Lee FK-H, Chan CC-L, Law C-K. Influence of CT contrast agent on dose calculation of intensity modulated radiation therapy plan for nasopharyngeal carcinoma. J Med Imaging Radiat Oncol, 53: 114-118.

[21] Zabel-du Bois A, Ackermann B, Hauswald H, Schramm O, Sroka-Perez G, Huber $P$, et al. Influence of intravenous contrast agent on dose calculation in 3-D treatment planning for radiosurgery of cerebral arteriovenous malformations. Strahlenther Onkol 2009;185:318-24.

[22] Sikora M, Muzik J, Söhn M, Weinmann M, Alber M. Monte Carlo vs. pencil beam based optimization of stereotactic lung IMRT. Radiat Oncol 2009;4:64.

[23] Antoch G, Kuehl H, Kanja J, Lauenstein TC, Schneemann H, Hauth E, et al. Dualmodality PET/CT scanning with negative oral contrast agent to avoid artifacts: introduction and evaluation. Radiology 2004;230:879-85.

[24] Brechtel K, Klein M, Vogel M, Mueller M, Aschoff P, Beyer T, et al. Optimized contrast-enhanced CT protocols for diagnostic whole-body 18F-FDG PET/CT: technical aspects of single-phase versus multiphase CT imaging. J Nucl Med 2006;47:470-6.

[25] Beyer T, Rosenbaum S, Veit P, Stattaus J, Müller SP, Difilippo FP, et al. Respiration artifacts in whole-body (18)F-FDG PET/CT studies with combined PET/ CT tomographs employing spiral CT technology with 1 to 16 detector rows. Eur J Nucl Med Mol Imaging 2005;32:1429-39.

[26] Kinahan PE, Hasegawa BH, Beyer T. X-ray-based attenuation correction for positron emission tomography/computed tomography scanners. Semin Nucl Med 2003;33:166-79.

[27] International Commission on Radiation Units and Measurements. ICRU report 62. Prescribing, recording, and reporting photon beam therapy (supplement to ICRU report 50). Bethesda, MD: ICRU; 1999.

[28] Erdi YE, Mawlawi O, Larson SM, Imbriaco M, Yeung H, Finn R, et al. Segmentation of lung lesion volume by adaptive positron emission tomography image thresholding. Cancer 1997;80(Suppl.):2505-9.

[29] Alber M, Birkner M, Laub M, Nüsslin F. Hyperion: an integrated IMRT planning tool. In: Schlegel W, Bortfeld T, editors. Proceedings of the XIII conference on the use of computers in radiation therapy. , Heidelberg: Springer; 2000. p. 46-8.

[30] Kurth J, Vogel H, Dunkelmann S, Hildebrandt G, Schümichen C. Nutzung des low-dose-CT der PET/CT für die Strahlentherapieplanung - Einfluss des CTProtokolls auf die CT-Elektronendichte-Konvertierung. Nuklearmedizin 2011;50:A51.

[31] Vanderstraeten B, Reynaert N, Paelinck L, Madani I, De Wagter C, De Gersem W, et al. Accuracy of patient dose calculation for lung IMRT: a comparison of Monte Carlo, convolution/superposition, and pencil beam. Med Phys 2006;33:3149-58.

[32] Hasenbalg F, Neuenschwander H, Mini R, Born EJ. Collapsed cone convolution and analytic anisotropic algorithm dose calculation compared to VMC ++ Monte Carlo simulations in clinical cases. Phys Med Biol 2007;52:3679-91.

[33] Beyer T, Antoch G, Müller S, Egelhof T, Freudenberg LS, Debatin J, et al. Acquisition protocol considerations for combined PET/CT imaging. J Nucl Med 2004;45(Suppl.1):25S-35S. 